ESAIM: M2AN 48 (2014) 1011-1027

DOI: $10.1051 / \mathrm{m} 2 \mathrm{an} / 2013131$
ESAIM: Mathematical Modelling and Numerical Analysis

www.esaim-m2an.org

\title{
RECONSTRUCTION OF THICKNESS VARIATION OF A DIELECTRIC COATING THROUGH THE GENERALIZED IMPEDANCE BOUNDARY CONDITIONS *
}

\author{
Birol AslanyüreK ${ }^{1}$ AND HÜLYA SAhintürk ${ }^{1}$
}

\begin{abstract}
We deal with an inverse scattering problem whose aim is to determine the thickness variation of a dielectric thin coating located on a conducting structure of unknown shape. The inverse scattering problem is solved through the application of the Generalized Impedance Boundary Conditions (GIBCs) which contain the thickness, curvature as well as material properties of the coating and they have been obtained in the previous work [B. Aslanyürek, H. Haddar and H. Şahintürk, Wave Motion 48 (2011) 681-700] up to the third order with respect to the thickness. After proving uniqueness results for the inverse problem, the required total field as well as its higher order derivatives appearing in the GIBCs are obtained by the analytical continuation of the measured data to the coating surface through the single layer potential representation. The resulting system of non-linear differential equations for the unknown coating thickness is solved iteratively via the Newton-Raphson method after expanding the thickness function in a series of exponentials. Through the simulations it has been shown that the approach is effective under the validity conditions of the GIBCs.
\end{abstract}

Mathematics Subject Classification. 78A25, 78A46, 78A45, 65N21.

Received July 3, 2012. Revised July 6, 2013.

Published online June 30, 2014.

\section{INTRODUCTION}

Generalized Impedance Boundary Conditions (GIBCs) which are used to model imperfectly conducting obstacles, scatterers with corrugated or rough surfaces, highly absorbing media, point defects or point conductors placed on a surface, dielectric resonators, conductive thin sheets, thin dielectric coatings, etc. simplify the mathematical and numerical complexities in the solution of scattering problems in electromagnetic theory and they have been widely studied in the open literature $[1,3,7,9-14,18-22,24-27,29-34]$. These studies mostly consist of deriving or dealing with GIBC expressions and using them in a direct scattering problem [9-12,14, 20-22, 25-27,30-32]. One can find also some applications of them to the inverse scattering problems for various geometries $[3,13,19]$.

Keywords and phrases. Generalized impedance boundary conditions, thin coatings, inverse scattering problems, single layer potential, Newton-Raphson method.

* B. Aslanyürek acknowledge the Scientific and Technological Research Council of Turkey (TUBITAK) for the financial support through Ph.D. scholarships.

1 Mathematical Engineering, Yildiz Technical University, Davutpasa, 34220 Istanbul, Turkey.

birolaslanyurek@yildiz.edu.tr; hsahin@yildiz.edu.tr 
Here we focus on the GIBCs defined on the surfaces of thin dielectric coatings in the concept of inverse scattering problems. In [5], the uniqueness and stability of inverse scattering problems under some proposed assumptions are investigated for the GIBCs defined on the surfaces of thin coatings, corrugated surfaces or highly absorbing media. In a sequel study to [5], the identification of a GIBC as well as the shape reconstruction for a obstacle is researched [4]. Also, a stable method is proposed to reconstruct the electromagnetic field in layered media having sufficiently small, constant thickness from overdetermined data on the outer boundary in [23].

The objective of this paper is to solve an inverse scattering problem whose aim is to determine the thickness of a thin dielectric coating located on a perfect magnetic conductor (PMC) by utilizing the GIBCs derived in [2]. In [2], several GIBCs expressions are derived for thin dielectric coatings with variable thickness, which is located on a perfect electric or magnetic conducting surface, and they are numerically validated through various numerical examples related to the direct scattering problem by using a finite element type method. The expressions of the GIBCs are obtained up to the third order with respect to the coating width for both Dirichlet and Neumann cases. Note that to use these GIBCs in the inverse scattering problems for the same configuration requires a substantially different approach and handling new difficulties due to the nature of the inverse scattering problems.

The GIBCs defined on a coating surface include geometrical and material properties of the coating as well as the total field and its high order normal and tangential derivatives. Here we first calculate these field values from measurements of the scattered field due to the coated object for one or several incident time harmonic plane waves at a fixed frequency. The scattered field is represented by a single-layer potential (SLP) [8] on a fictitious surface that is close to the surface of the coating and the density of the single-layer potential is obtained by solving the resulting ill-posed integral equation of the first kind through the truncated singular value decomposition (TSVD) regularization [8]. Then the required total fields and their derivatives are obtained on the coating surface without tackling difficulty of singularity. By substituting the obtained fields into the expressions of the GIBCs, the problem is reduced to the solutions of systems of differential equations for orders 1,2 and 3. Actually this is a 1D coefficient identification problem and there are divers studies in the literature $[16,17]$. In this paper, in order to solve these problems, we seek the width of the coating as a series in terms of exponential functions. Thus, the problem is transformed to the solution of a system of linear equations for case of order 1 and a system of nonlinear equations for cases order 2 and 3. The nonlinear systems of equations are solved numerically by means of a well-known Newton-Raphson Method. The method is tested by considering several coating configurations and it has been shown that it yields quite satisfactory reconstructions for the thicknesses in the order of $\lambda / 10$, where $\lambda$ is the wavelength of the incident wave.

It should be noted that although the method is developed for the coatings located on a perfect magnetic conductor, it can be easily applied to the perfect electric case which is much more simpler. Besides, the method is presented as the thickness reconstruction of the coating but it is also equivalent to shape reconstruction of a perfect magnetic conductor located in a dielectric object.

The document is organized as follows. Section 2 is dedicated to introduce the original inverse scattering problem for thickness reconstruction of a coating, and to give its equivalent model in terms of the GIBCs. After proving uniqueness results for the inverse problem in Section 2.1, the equivalent problem is solved in two steps: obtaining the total field and its partial derivatives on the surface of the coating via the SLP in Section 2.2 and calculating the width of the coating from the GIBCs of orders 1, 2 and 3 in Section 2.3. Finally, the effectiveness of the approach given here is illustrated through several numerical examples in Section 3. A time factor $\mathrm{e}^{-i \omega t}$ is assumed and omitted throughout the paper.

\section{Formulation OF THE PROBLEM}

Consider the inverse electromagnetic scattering problem illustrated in Figure 1. In this configuration a homogeneous, lossy, non-magnetic dielectric material coating $\Omega_{+}$whose dielectric permittivity $\varepsilon_{1}$ and conductivity $\sigma_{1}$ is located on a perfect magnetic conducting (PMC) cylinder with boundary $\Gamma^{\delta}$. The background medium 


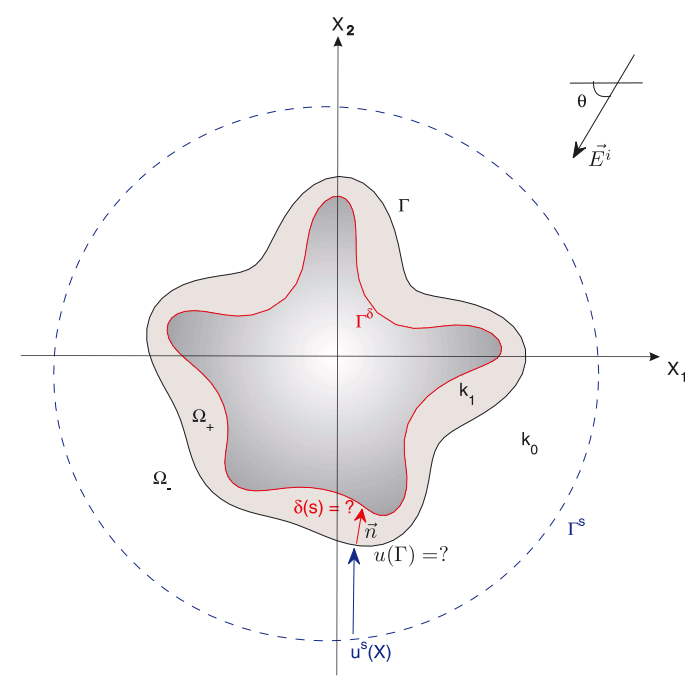

FiguRE 1. Geometry of the inverse problem.

represented by $\Omega_{-}$is the free-space with dielectric permittivity $\varepsilon_{0}$ and magnetic permeability $\mu_{0}$. The inverse scattering problem for this configuration which will be dealt with here is to determine the thickness variation $\delta$ of the coating by illuminating the coated cylinder by a number of electromagnetic waves and measuring the scattered field on a circular region $\Gamma^{s}$ (see Fig. 1). The geometry of the outer boundary of the coating $\Gamma$, which is assumed to be at least twice continuously differentiable, as well as coating material are assumed to be known.

Let us assume that the coated cylinder is located along to $x_{3}$ axis and is illuminated by a TM polarized time-harmonic plane wave whose electric field vector $\vec{E}^{i}$ is always parallel to the $x_{3}$ axis, namely;

$$
\overrightarrow{E^{i}}=\left(0,0, u^{i}(x)\right)
$$

with

$$
u^{i}(x)=\mathrm{e}^{-i \kappa_{0} x \cdot d}
$$

where $d=(\cos \theta, \sin \theta)$ is the propagation direction with incidence angle $\theta$ and $\kappa_{0}$ is the wave number of freespace. In this case, the total electric field $\vec{E}$ is also in the form $\vec{E}=\left(0,0, u^{\delta}(x)\right)$ and the problem is reduced to a scalar one in $\mathbb{R}^{2}$ for the field function $u^{\delta}$. The total field $u^{\delta}$ satisfies the reduced wave equation

$$
\Delta u^{\delta}+\kappa^{2} u^{\delta}=0 \quad \text { in } \Omega_{-} \cup \Omega_{+}
$$

with the Neumann boundary condition

$$
\frac{\partial u^{\delta}}{\partial n^{\delta}}=0 \quad \text { on } \Gamma^{\delta}
$$

where

$$
\kappa^{2}(x)= \begin{cases}\kappa_{0}^{2}=\omega^{2} \varepsilon_{0} \mu_{0}, & \text { in } \Omega_{-}, \\ \kappa_{1}^{2}=\omega^{2} \varepsilon_{1} \mu_{0}+i \omega \sigma_{1} \mu_{0}, & \text { in } \Omega_{+},\end{cases}
$$

is the square of piecewise constant wave number as well as $\vec{n}^{\delta}$ is the unitary normal vector directed to the interior of $\Omega_{+}$on $\Gamma^{\delta}$.

Besides of Neumann boundary condition, the scattering problem has continuity conditions, namely, the total field $u^{\delta}$ and its normal derivative $\frac{\partial u^{\delta}}{\partial n}$ are continues on $\Gamma$, and Sommerfeld radiation condition

$$
\lim _{r \rightarrow \infty} \sqrt{r}\left(\frac{\partial u^{s}}{\partial r}(x)-i \kappa u^{s}(x)\right)=0, \quad r=|x|
$$


where the scattered field, $u^{s}$, is defined as

$$
u^{s}=u^{\delta}-u^{i} \quad \text { in } \Omega_{-} .
$$

The scattering problem whose formulation given above can be converted into the following form through the GIBCs. Namely, we first introduce the Neumann impedance operator $N^{\delta}$ which approximately satisfies

$$
\frac{\partial u^{\delta}}{\partial n}+N^{\delta} u^{\delta}=0 \quad \text { on } \Gamma
$$

where $n$ is directed to the interior of $\Omega_{+}$. An impedance operator suited to the problem given here are derived in [2] up to the third order with respect to the coating width. These operators, including information related to geometric and electromagnetic properties of the coating, are given as

$$
\begin{aligned}
N^{\delta, 1}= & -\frac{\partial}{\partial s} \delta \frac{\partial}{\partial s}-\delta \kappa_{1}^{2} \\
N^{\delta, 2}= & -\frac{\partial}{\partial s}\left(\delta-\frac{1}{2} \delta^{2} c\right) \frac{\partial}{\partial s}-\left(\delta+\frac{1}{2} \delta^{2} c\right) \kappa_{1}^{2} \\
N^{\delta, 3}= & -\frac{\partial}{\partial s}\left(\delta-\frac{1}{2} \delta^{2} c+\frac{1}{3} \delta^{3} c^{2}+\frac{2}{3} \kappa_{1}^{2} \delta^{3}+\frac{1}{2} \delta^{2} \delta^{\prime \prime}\right) \frac{\partial}{\partial s} \\
& -\left(\delta+\frac{1}{2} \delta^{2} c+\frac{1}{3} \kappa_{1}^{2} \delta^{3}+\frac{1}{2} \delta^{2} \delta^{\prime \prime}+\delta\left(\delta^{\prime}\right)^{2}\right) \kappa_{1}^{2}-\frac{1}{3} \frac{\partial^{2}}{\partial s^{2}} \delta^{3}(s) \frac{\partial^{2}}{\partial s^{2}}
\end{aligned}
$$

for orders 1, 2 and 3, respectively. Here the boundary $\Gamma$ is parameterized in terms of the curvilinear abscissa $s$ as $s \mapsto x_{\Gamma}(s)=\left(x_{1}(s), x_{2}(s)\right)^{t}, s \in\left[0, L\left[\right.\right.$, with $\left|\mathrm{d} x_{\Gamma}(s) / \mathrm{d} s\right|=1$, where $L$ is the length of $\Gamma$. This parametrization defines a counter-clockwise orientation. The curvature of the boundary $\Gamma$ is defined as $c(s)=\vec{\tau}(s) \cdot \mathrm{d} \vec{n}(s) / \mathrm{d} s$ where $\vec{\tau}(s)=\mathrm{d} x_{\Gamma}(s) / \mathrm{d} s$ is a unitary vector tangential to $\Gamma$ at $x_{\Gamma}(s)$. The width of the coating, $\delta$, is defined clearly as

$$
\delta(s)=\operatorname{dist}\left(x_{\Gamma}(s), \Gamma^{\delta}\right) \quad s \in[0, L[
$$

and is assumed to be sufficiently regular and small, so that

$$
s \rightarrow x_{\Gamma}^{\delta}(s):=x_{\Gamma}(s)+\delta(s) \vec{n}(s)
$$

defines a global and injective parametrization of $\Gamma^{\delta}$ in terms of $s \in\left[0, L\left[\right.\right.$. In $(2.11), \delta^{\prime}$ and $\delta^{\prime \prime}$ represent respectively the first and second derivative of $\delta$ with respect to $s$. Note that although the boundary $\Gamma$ is assumed to be at least twice continuously differentiable, the third order boundary condition contains higher order derivatives. We will not make any assumption on this, yet we will do formal computations. We refer the readers to [2] for more information related to the GIBCs handled here.

Thus, after introducing the GIBCs on the boundary $\Gamma$, the scattering problem given by (2.1)-(2.7) can now be reformulated as follows: If the total field of the equivalent problem denoted by $u$, it satisfies the reduced wave equation

$$
\Delta u+\kappa^{2} u=0 \quad \text { in } \Omega_{-}
$$

with the GIBC

$$
\frac{\partial u}{\partial n}+N^{\delta} u=0 \quad \text { on } \Gamma
$$

and the Sommerfeld Boundary Condition given in (2.6). In this case, the inverse scattering problem mentioned above can be stated as solving the unknown thickness $\delta(s)$ from the boundary condition (2.14). Note that in order to solve $\delta(s)$ from (2.14), it is required to know the total field $u$ and its derivatives with respect to $n$ and $s$ on $\Gamma$. On the other hand one has only the values of the total field on the measurement surface $\Gamma^{s}$. In the following, after the investigation of the uniqueness for the inverse problem formulated above, the next subsections are dedicated to the calculation of $u, \frac{\partial u}{\partial s}, \frac{\partial u}{\partial n}$ on $\Gamma$ from the measured data on $\Gamma^{s}$ and the determining of $\delta$ from the GIBCs expressions, respectively. 
Note that, the formal error that occurs due to difference between equivalent problems satisfies

$$
\left\|u^{\delta}-u\right\|_{\Omega_{-}} \leq a \delta^{m+1}
$$

where the constant $a$ does not depend on $\delta$ and $m$ is the order of the GIBCs [2,9].

\subsection{Uniqueness of the solution of the inverse problem}

In this section it will be provided uniqueness results concerning the reconstruction of the thickness $\delta(s)$ from the GIBCs for given values of the scattered field. The proofs will be given for the individual GIBCs which are defined by means of the operators given in (2.9)-(2.11). For the first and second order GIBCs we refer the readers to [4]. In that work, under Assumption 2.1 given below, a uniqueness proof has been presented for a GIBC which can be written in the scalar form

$$
\frac{\partial u}{\partial n}-\frac{\partial}{\partial s} \mu \frac{\partial}{\partial s} u-\lambda u=0 \quad \text { on } \Gamma
$$

for the unit normal to $\Gamma$ directed to the interior of $\Omega_{+}$. Here $\lambda$ and $\mu$ are complex valued functions and satisfy $\lambda \in C^{0}(\Gamma)$ and $\mu \in C^{1}(\Gamma)$.

Assumption 2.1. The coefficients $(\lambda, \mu) \in\left(L^{\infty}(\Gamma)\right)^{2}$ are such that

$$
\operatorname{Im}(\lambda) \geq 0, \operatorname{Im}(\mu) \leq 0
$$

and there exist $d>0$ such that

$$
\operatorname{Re}(\mu) \geq d
$$

Let $u$ be the field on boundary $\Gamma$ and $\left(\lambda_{1}, \mu_{1}\right)$ and $\left(\lambda_{2}, \mu_{2}\right)$ be two solutions. The proof given in [4] consists in proving that $\left(\lambda_{1}, \mu_{1}\right)=\left(\lambda_{2}, \mu_{2}\right)$. By setting $\lambda=\lambda_{1}-\lambda_{2}$ and $\mu=\mu_{1}-\mu_{2}$, from the boundary condition (2.16) it follows that

$$
\frac{\partial}{\partial s} \mu \frac{\partial}{\partial s} u+\lambda u=0 \quad \text { on } \Gamma
$$

By using the inner product defined as

$$
\left\langle\frac{\partial}{\partial s} \mu \frac{\partial}{\partial s} u, v\right\rangle_{H^{-1}(\Gamma), H^{1}(\Gamma)}=-\int_{\Gamma} \mu \frac{\partial u}{\partial s} \frac{\partial v}{\partial s} \mathrm{~d} s, \quad \forall v \in H^{1}(\Gamma),
$$

it can be shown that

$$
\left\langle u, \frac{\partial}{\partial s} \mu \frac{\partial}{\partial s} \phi+\lambda \phi\right\rangle_{H^{1}(\Gamma), H^{-1}(\Gamma)}=0
$$

for some $\phi \in H^{1}(\Gamma)$. Then, by the help of Lemma 3.3 given in [4], one can write

$$
\frac{\partial}{\partial s} \mu \frac{\partial}{\partial s} \phi+\lambda \phi=0 \quad \text { on } \Gamma, \quad \forall \phi \in H^{1}(\Gamma) .
$$

Choosing $\phi=1$ in (2.20) leads to $\lambda=0$. Then, using of energy methods for (2.20), one can also obtain $\mu=0$ and complete the proof as elaborated in [4].

Now we discuss uniqueness for the GIBC problem dealt in this paper by the help of above procedure.

For the first order GIBC. By choosing $\lambda=\delta \kappa_{1}^{2}$ and $\mu=\delta$, which are satisfy Assumption 2.1, the GIBC for the first order becomes identical to that given in (2.16), which has a unique solution.

For the second order GIBC. In a similar way, it can be easily shown that (2.14) with (2.10) and (2.16) are identical for $\lambda=\left(\delta+\frac{1}{2} \delta^{2} c\right) \kappa_{1}^{2}$ and $\mu=\left(\delta-\frac{1}{2} \delta^{2} c\right)$. Reminding that $\delta>\frac{1}{2} \delta^{2} c$ due to the asymptotic approach 
used in [2], it is clearly seen that $\left(\delta+\frac{1}{2} \delta^{2} c\right) \kappa_{1}^{2}=0$ and $\left(\delta-\frac{1}{2} \delta^{2} c\right)=0$ which leads to $\delta=0$. This result completes the proof by assuming that $\delta=\delta_{1}-\delta_{2}$ where $\delta_{1}$ and $\delta_{2}$ are two solutions of the inverse problem.

For the third order GIBC. Although the form of the third order GIBC is different than that of (2.16), we can use the similar procedure to prove the uniqueness of the solution. Under the assumption that $\delta_{1}$ and $\delta_{2}$ are two solutions of the inverse problem, for $\delta=\delta_{1}-\delta_{2}$, the third order GIBC can be written as

$$
\frac{\partial^{2}}{\partial s^{2}} \varphi \frac{\partial^{2}}{\partial s^{2}} u+\frac{\partial}{\partial s} \mu \frac{\partial}{\partial s} u+\lambda u=0 \quad \text { on } \Gamma
$$

where

$$
\begin{gathered}
\varphi=\frac{1}{3} \delta^{3}, \\
\mu=\delta-\frac{1}{2} \delta^{2} c+\frac{1}{3} \delta^{3} c^{2}+\frac{2}{3} \kappa_{1}^{2} \delta^{3}+\frac{1}{2} \delta^{2} \delta^{\prime \prime}, \\
\lambda=\left(\delta+\frac{1}{2} \delta^{2} c+\frac{1}{3} \kappa_{1}^{2} \delta^{3}+\frac{1}{2} \delta^{2} \delta^{\prime \prime}+\delta\left(\delta^{\prime}\right)^{2}\right) \kappa_{1}^{2} .
\end{gathered}
$$

By following the derivation procedure of (2.20) from (2.17) above, one gets

$$
\frac{\partial^{2}}{\partial s^{2}} \varphi \frac{\partial^{2}}{\partial s^{2}} \phi+\frac{\partial}{\partial s} \mu \frac{\partial}{\partial s} \phi+\lambda \phi=0 \quad \text { on } \Gamma, \forall \phi \in H^{1}(\Gamma) .
$$

Then substitution of $\phi=1, \phi=s, \phi=s^{2}, \phi=s^{3}$ and $\phi=s^{4}$ into (2.22) yields to get

$$
\begin{gathered}
\lambda=0, \\
\frac{\partial \mu}{\partial s}=0, \\
\frac{\partial^{2} \varphi}{\partial s^{2}}+\mu=0, \\
\frac{\partial \varphi}{\partial s}=0, \\
\varphi=0,
\end{gathered}
$$

respectively, which gives $\delta=0$.

\subsection{Derivation of the total field and its derivatives on the impedance surface}

In order to derive the total field and its derivatives with respect to the curvilinear abscissa $\mathrm{s}$ and the inner unitary normal vector $\mathrm{n}$ on the coating surface $\Gamma$, we represent the scattered field $u^{s}$ as a SLP

$$
u^{s}(X)=\int_{\Gamma} G(X ; Y) \Psi(Y) d s(Y) \quad X \in \Gamma^{s}, Y \in \Gamma
$$

with unknown density function $\Psi$ on $\Gamma[8]$. Here $\mathrm{G}$ is the Green's function of free-space given by

$$
G(X ; Y)=\frac{i}{4} H_{0}^{(1)}\left(k_{0}|X-Y|\right)
$$

where $H_{0}^{(1)}$ denotes the Hankel function of the first kind and order 0 . For the given values of the scattered field on $\Gamma^{s}$, one needs to solve (2.23) for the unknown density $\Psi$ and reuse it to calculate the scattered field on $\Gamma$. 


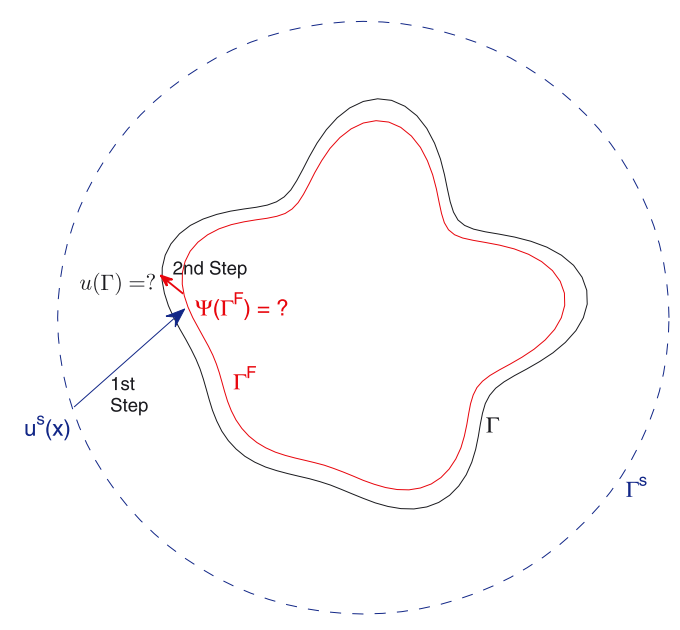

Figure 2. Defining density $\Psi$ on $\Gamma^{F}$ instead of $\Gamma$.

As seen clearly, (2.23) has singularity at $X=Y$ because of free-space Green's function. One can tackle this difficulty by using the Nyström method [8]. Here, we apply a more practical approach based on defining density function on a fictitious surface $\Gamma^{F}$ selected a bit closer to the origin instead of $\Gamma$ as shown Figure 2 (see [6]). Thus, modifying the SLP given in (2.23), one can write it in symbolic form as

$$
A \Psi=u^{s}(X) \quad X \in \Gamma^{s}
$$

where the integral operator $\mathrm{A}$ is given by

$$
A \Psi=\int_{\Gamma^{F}} G(X ; Y) \Psi(Y) \mathrm{d} s(Y) \quad X \in \Gamma^{s}, Y \in \Gamma^{F} .
$$

Equation (2.25) is ill-posed since the operator A has an analytic kernel. That's why some kind of regularization such as TSVD [8] has to be applied. Considering the compact linear operator A with singular system $\left(\sigma_{n}, \psi_{n}, v_{n}\right), n \in \mathbb{N}$, the regularized solution is expressed by TSVD inversion formula

$$
\Psi=\sum_{n=1}^{N} \frac{1}{\sigma_{n}}\left\langle u^{s}, v_{n}\right\rangle \psi_{n}
$$

where $\langle$,$\rangle denotes the inner product on \mathrm{L}^{2}[8]$. Here the truncation parameter $N$ is assigned according to the measurement noise.

Once the density function $\Psi$ is obtained by (2.27), the total field

$$
u(X)=u^{i}(X)+\int_{\Gamma^{F}} G(X ; Y) \Psi(Y) \mathrm{d} s(Y) \quad X \in \Gamma, Y \in \Gamma^{F},
$$

the derivative of the total field with respect to $\mathrm{s}$

$$
\frac{\partial u(X)}{\partial s}=\frac{\partial u^{i}(X)}{\partial s}+\int_{\Gamma^{F}} \frac{\partial G(X ; Y)}{\partial s} \Psi(Y) \mathrm{d} s(Y) \quad X \in \Gamma, Y \in \Gamma^{F},
$$

and the derivative of the total field with respect to $\mathrm{n}$

$$
\frac{\partial u(X)}{\partial n}=\frac{\partial u^{i}(X)}{\partial n}+\int_{\Gamma^{F}} \frac{\partial G(X ; Y)}{\partial n} \Psi(Y) \mathrm{d} s(Y) \quad X \in \Gamma, Y \in \Gamma^{F}
$$

can be calculated. 
Note that here we only give the explicit expressions of the total field and its first order derivatives. On the other hand, the Neumann operators given in (2.9), (2.10) and (2.11) include also the higher order derivatives with respect to $s$. In the solution of the inverse scattering problem below, we will first rearrange the equations to include only first order derivatives, thus, (2.28), (2.29) and (2.30) will be enough to get the required solutions.

\subsection{Reconstruction of thickness variation}

Let us first substitute the Neumann operators given in (2.9), (2.10) and (2.11) into the boundary condition (2.14), and multiply the both sides of the resulting equations by $v_{m}=\mathrm{e}^{-i \frac{2 \pi s}{|T|} m}$ and then integrate them over $\Gamma$ to obtain

$$
\begin{aligned}
\int_{\Gamma} \frac{\partial u}{\partial n} v_{m} \mathrm{~d} s= & \int_{\Gamma}\left(\frac{\partial}{\partial s} \delta \frac{\partial}{\partial s} u+\delta \kappa_{1}^{2} u\right) v_{m} \mathrm{~d} s, \quad m=0, \pm 1, \ldots, \pm M, \\
\int_{\Gamma} \frac{\partial u}{\partial n} v_{m} \mathrm{~d} s= & \int_{\Gamma}\left(\frac{\partial}{\partial s}\left(\delta-\frac{1}{2} \delta^{2} c\right) \frac{\partial}{\partial s} u+\left(\delta+\frac{1}{2} \delta^{2} c\right) \kappa_{1}^{2} u\right) v_{m} \mathrm{~d} s \\
m & =0, \pm 1, \ldots, \pm M, \\
\int_{\Gamma} \frac{\partial u}{\partial n} v_{m} \mathrm{~d} s= & \int_{\Gamma}\left(\frac{\partial}{\partial s}\left(\delta-\frac{1}{2} \delta^{2} c+\frac{1}{3} \delta^{3} c^{2}+\frac{2}{3} \kappa_{1}^{2} \delta^{3}+\frac{1}{2} \delta^{2} \frac{\partial^{2} \delta}{\partial s^{2}}\right) \frac{\partial}{\partial s} u\right) v_{m} \mathrm{~d} s \\
& +\int_{\Gamma}\left(\delta+\frac{1}{2} \delta^{2} c+\frac{1}{3} \kappa_{1}^{2} \delta^{3}+\frac{1}{2} \delta^{2} \frac{\partial^{2} \delta}{\partial s^{2}}+\delta\left(\frac{\partial \delta}{\partial s}\right)^{2}\right) \kappa_{1}^{2} u v_{m} \mathrm{~d} s \\
& +\frac{1}{3} \int_{\Gamma}\left(\frac{\partial^{2}}{\partial s^{2}} \delta^{3} \frac{\partial^{2}}{\partial s^{2}} u\right) v_{m} \mathrm{~d} s, \quad m=0, \pm 1, \ldots, \pm M
\end{aligned}
$$

for orders 1, 2 and 3, respectively. As clearly be seen from (2.31), (2.32) and (2.33) the resulting expressions contain higher order derivatives of the total field with respect to $s$. In order to reduce the numerical difficulties due to the calculation of these higher order derivatives of the total field in the solution of the inverse scattering problem, it will be convenient to write them in terms of first order derivatives only. This can be achieved by successive partial integrations of the terms containing higher order derivatives of $u$ in (2.31), (2.32) and (2.33). Then one has,

$$
\begin{aligned}
\int_{\Gamma} \frac{\partial u}{\partial n} v_{m} \mathrm{~d} s= & \int_{\Gamma} \delta\left(\kappa_{1}^{2} u v_{m}-\frac{\partial u}{\partial s} \frac{\partial v_{m}}{\partial s}\right) \mathrm{d} s, \quad m=0, \pm 1, \ldots, \pm M \\
\int_{\Gamma} \frac{\partial u}{\partial n} v_{m} \mathrm{~d} s= & \int_{\Gamma} \delta\left(\kappa_{1}^{2} u v_{m}-\frac{\partial u}{\partial s} \frac{\partial v_{m}}{\partial s}\right) \mathrm{d} s+\frac{1}{2} \int_{\Gamma} \delta^{2} c\left(\kappa_{1}^{2} u v_{m}+\frac{\partial u}{\partial s} \frac{\partial v_{m}}{\partial s}\right) \mathrm{d} s \\
& m=0, \pm 1, \ldots, \pm M \\
\int_{\Gamma} \frac{\partial u}{\partial n} v_{m} \mathrm{~d} s= & \int_{\Gamma} \delta\left(\kappa_{1}^{2} u v_{m}-\frac{\partial u}{\partial s} \frac{\partial v_{m}}{\partial s}\right) \mathrm{d} s+\frac{1}{2} \int_{\Gamma} \delta^{2} c\left(\kappa_{1}^{2} u v_{m}+\frac{\partial u}{\partial s} \frac{\partial v_{m}}{\partial s}\right) \mathrm{d} s \\
& +\frac{1}{3} \int_{\Gamma} \delta^{3}\left(\kappa_{1}^{4} u v_{m}-c^{2} \frac{\partial u}{\partial s} \frac{\partial v_{m}}{\partial s}-2 \kappa_{1}^{2} \frac{\partial u}{\partial s} \frac{\partial v_{m}}{\partial s}-\frac{\partial u}{\partial s} \frac{\partial^{3} v_{m}}{\partial s^{3}}\right) \mathrm{d} s \\
& -\int_{\Gamma} \delta^{2} \frac{\partial \delta}{\partial s} \frac{\partial u}{\partial s} \frac{\partial^{2} v_{m}}{\partial s^{2}} \mathrm{~d} s+\frac{1}{2} \int_{\Gamma} \delta^{2} \frac{\partial^{2} \delta}{\partial s^{2}}\left(\kappa_{1}^{2} u v_{m}-\frac{\partial u}{\partial s} \frac{\partial v_{m}}{\partial s}\right) \mathrm{d} s \\
& +\int_{\Gamma} \delta\left(\frac{\partial \delta}{\partial s}\right)^{2} \kappa_{1}^{2} u v_{m} \mathrm{~d} s, \quad m=0, \pm 1, \ldots, \pm M
\end{aligned}
$$

for orders 1, 2 and 3, respectively. The inverse scattering problem is reformulated as the solution of (2.34), (2.35) and (2.36), which are nothing but integro-differential equations for the unknown thickness function $\delta$. In order to solve $\delta$ from these systems of equations, we first represent the thickness function $\delta(s)$ as a series in terms of 
exponential functions as follows:

$$
\delta(s)=\sum_{k=-K}^{K} \alpha_{k} \phi_{k}(s)
$$

where $\alpha_{k}, \quad k=0, \pm 1, \ldots, \pm K$, are unknown coefficients to be determined while the functions $\phi_{k}$ 's, which are $L$-periodic on $[0, \mathrm{~L}[$, are given as

$$
\phi_{k}(s)=\mathrm{e}^{i \frac{2 \pi s}{|\Gamma|} k}
$$

for $k=0, \pm 1, \ldots, \pm K$. Note that, with the representation in (2.37), any derivative of $\delta(s)$ with respect to $s$ can be expressed analytically. Then substitution of (2.37) into (2.34), (2.35) and (2.36) results in

$$
\begin{aligned}
& \int_{\Gamma} \frac{\partial u}{\partial n} v_{m} \mathrm{~d} s=\sum_{k=-K}^{K} \alpha_{k} \int_{\Gamma} \phi_{k}\left(\kappa_{1}^{2} u v_{m}-\frac{\partial u}{\partial s} \frac{\partial v_{m}}{\partial s}\right) \mathrm{d} s, \quad m=0, \pm 1, \ldots, \pm M, \\
& \int_{\Gamma} \frac{\partial u}{\partial n} v_{m} \mathrm{~d} s=\sum_{k=-K}^{K} \alpha_{k} \int_{\Gamma} \phi_{k}\left(\kappa_{1}^{2} u v_{m}-\frac{\partial u}{\partial s} \frac{\partial v_{m}}{\partial s}\right) \mathrm{d} s \\
& +\frac{1}{2} \int_{\Gamma}\left(\sum_{k=-K}^{K} \alpha_{k} \phi_{k}\right)^{2} c\left(\kappa_{1}^{2} u v_{m}+\frac{\partial u}{\partial s} \frac{\partial v_{m}}{\partial s}\right) \mathrm{d} s, \\
& m=0, \pm 1, \ldots, \pm M \text {, } \\
& \int_{\Gamma} \frac{\partial u}{\partial n} v_{m} \mathrm{~d} s=\sum_{k=-K}^{K} \alpha_{k} \int_{\Gamma} \phi_{k}\left(\kappa_{1}^{2} u v_{m}-\frac{\partial u}{\partial s} \frac{\partial v_{m}}{\partial s}\right) \mathrm{d} s \\
& +\frac{1}{2} \int_{\Gamma}\left(\sum_{k=-K}^{K} \alpha_{k} \phi_{k}\right)^{2} c\left(\kappa_{1}^{2} u v_{m}+\frac{\partial u}{\partial s} \frac{\partial v_{m}}{\partial s}\right) \mathrm{d} s \\
& +\frac{1}{3} \int_{\Gamma}\left(\sum_{k=-K}^{K} \alpha_{k} \phi_{k}\right)^{3}\left(\kappa_{1}^{4} u v_{m}-c^{2} \frac{\partial u}{\partial s} \frac{\partial v_{m}}{\partial s}-2 \kappa_{1}^{2} \frac{\partial u}{\partial s} \frac{\partial v_{m}}{\partial s}-\frac{\partial u}{\partial s} \frac{\partial^{3} v_{m}}{\partial s^{3}}\right) \mathrm{d} s \\
& -\int_{\Gamma}\left(\sum_{k=-K}^{K} \alpha_{k} \phi_{k}\right)^{2}\left(\sum_{k=-K}^{K} \alpha_{k} \frac{\partial \phi_{k}}{\partial s}\right) \frac{\partial u}{\partial s} \frac{\partial^{2} v_{m}}{\partial s^{2}} \mathrm{~d} s \\
& +\frac{1}{2} \int_{\Gamma}\left(\sum_{k=-K}^{K} \alpha_{k} \phi_{k}\right)^{2}\left(\sum_{k=-K}^{K} \alpha_{k} \frac{\partial^{2} \phi_{k}}{\partial s^{2}}\right)\left(\kappa_{1}^{2} u v_{m}-\frac{\partial u}{\partial s} \frac{\partial v_{m}}{\partial s}\right) \mathrm{d} s \\
& +\int_{\Gamma}\left(\sum_{k=-K}^{K} \alpha_{k} \phi_{k}\right)\left(\sum_{k=-K}^{K} \alpha_{k} \frac{\partial \phi_{k}}{\partial s}\right)^{2} \kappa_{1}^{2} u v_{m} \mathrm{~d} s, \quad m=0, \pm 1, \ldots, \pm M
\end{aligned}
$$

for orders 1, 2 and 3, respectively. For a single illumination, (2.39), (2.40) and (2.41) severally constitute a system of algebraic equations with dimensions $(2 M+1) \times(2 K+1)$. Note that for the GIBC of order $1,(2.39)$ is a linear algebraic system and can be solved easily through matrix inversion in the least square sense.

On the other hand, (2.40) and (2.41) are non-linear algebraic systems for the coefficients $\alpha_{k}$. Here we propose a Newton-Raphson type iterative method for the solution of these equations.

Consider the continuously differentiable function $F: \mathbb{C}^{2 K+1} \rightarrow \mathbb{C}^{2 M+1}$ which represents one system of nonlinear equations in (2.40) or (2.41). Then, the iterative solution in Newton sense is given by,

$$
J_{F}\left(\alpha^{(t)}\right)\left(\alpha^{(t+1)}-\alpha^{(t)}\right)=-F\left(\alpha^{(t)}\right)
$$


with

$$
\alpha=\left[\begin{array}{c}
\alpha_{-K} \\
\vdots \\
0 \\
\vdots \\
\alpha_{K}
\end{array}\right] .
$$

where $t$ denotes the $t$ th iteration. The Jacobian matrix in (2.42) is denoted by

$$
J_{F}=\frac{\partial\left(F^{(-M)}, \ldots, F^{(m)}, \ldots, F^{(M)}\right)}{\partial\left(\alpha_{-K}, \ldots, \alpha_{k}, \ldots, \alpha_{K}\right)}
$$

where

$$
\begin{aligned}
\frac{\partial F^{(m)}}{\partial \alpha_{k}}= & \int_{\Gamma} \phi_{k}\left(\kappa_{1}^{2} u v_{m}-\frac{\partial u}{\partial s} \frac{\partial v_{m}}{\partial s}\right) \mathrm{d} s \\
& +\int_{\Gamma} \delta \phi_{k} c\left(\kappa_{1}^{2} u v_{m}+\frac{\partial u}{\partial s} \frac{\partial v_{m}}{\partial s}\right) \mathrm{d} s \quad \text { for order } 2 \\
\frac{\partial F^{(m)}}{\partial \alpha_{k}}= & \int_{\Gamma} \phi_{k}\left(\kappa_{1}^{2} u v_{m}-\frac{\partial u}{\partial s} \frac{\partial v_{m}}{\partial s}\right) \mathrm{d} s+\int_{\Gamma} \delta \phi_{k} c\left(\kappa_{1}^{2} u v_{m}+\frac{\partial u}{\partial s} \frac{\partial v_{m}}{\partial s}\right) \mathrm{d} s \\
& +\int_{\Gamma} \delta^{2} \phi_{k}\left(\kappa_{1}^{4} u v_{m}-c^{2} \frac{\partial u}{\partial s} \frac{\partial v_{m}}{\partial s}-2 \kappa_{1}^{2} \frac{\partial u}{\partial s} \frac{\partial v_{m}}{\partial s}-\frac{\partial u}{\partial s} \frac{\partial^{3} v_{m}}{\partial s^{3}}\right) \mathrm{d} s \\
& -\int_{\Gamma}\left(2 \delta \frac{\partial \delta}{\partial s} \phi_{k}+\delta^{2} \frac{\partial \phi_{k}}{\partial s}\right) \frac{\partial u}{\partial s} \frac{\partial^{2} v_{m}}{\partial s^{2}} \\
& +\frac{1}{2} \int_{\Gamma}\left(2 \delta \frac{\partial^{2} \delta}{\partial s^{2}} \phi_{k}+\delta^{2} \frac{\partial^{2} \phi_{k}}{\partial s^{2}}\right)\left(\kappa_{1}^{2} u v_{m}-\frac{\partial u}{\partial s} \frac{\partial v_{m}}{\partial s}\right) \mathrm{d} s \\
& +\int_{\Gamma}\left(\phi_{k}\left(\frac{\partial \delta}{\partial s}\right)^{2}+2 \delta \frac{\partial \delta}{\partial s} \frac{\partial \phi_{k}}{\partial s}\right) \kappa_{1}^{2} u v_{m} \mathrm{~d} s \quad \text { for order } 3 .
\end{aligned}
$$

The first initial guess $\alpha^{(0)}$ is critical for the Newton algorithm to converge. Fortunately, $\delta$ calculated from the linear problem of the GIBC of order 1 is probably sufficient as a first initial guess for the non-linear ones.

The system of non-linear equations (2.42) can be solved through the inverse of the Jacobian matrix, if the Jacobian matrix is square. If the system becomes overdetermined, one can solve this system by computing the generalized least squares inverse [28] of the non-square Jacobian matrix or apply the least square method.

\section{Numerical Results}

We now present some numerical results to demonstrate the effectiveness and applicability of the reconstruction approach given in the previous section. In all examples, the coated object is assumed to be located in free space and is illuminated by 6 plane waves with incident angles $\theta=\frac{j \pi}{3}(j=0,1, \ldots, 5)$ unless otherwise stated. By solving the direct scattering problem related to the original problem mentioned in Section 2 via the Finite Element Method (FEM) [2], the scattered field data are synthetically generated on $\Gamma^{S}$ at 100 points. A $10 \%$ random noise, namely $n_{r}\left|u^{s}\right| \mathrm{e}^{2 i r \pi}$ with the noise level $n_{r}=10 \%$ and a random number $r$ between 0 and 1 , is added to each scattered field value $u^{s}$.

The first step of the approach requires to calculate the total field $u$ and its derivatives $\frac{\partial u}{\partial s}$ and $\frac{\partial u}{\partial n}$ on the coating surface $\Gamma$ through the measured scattered data. To this aim, the SLP representation is used and the density function $\Psi$ is defined on $\Gamma^{F}$ whose parametric equation is given by $X_{\Gamma^{F}}=0.9 X_{\Gamma}$ for all simulations. In this procedure a regularization is applied by representation $\Psi$ in terms of the TSVD and the truncation parameter $N$ 

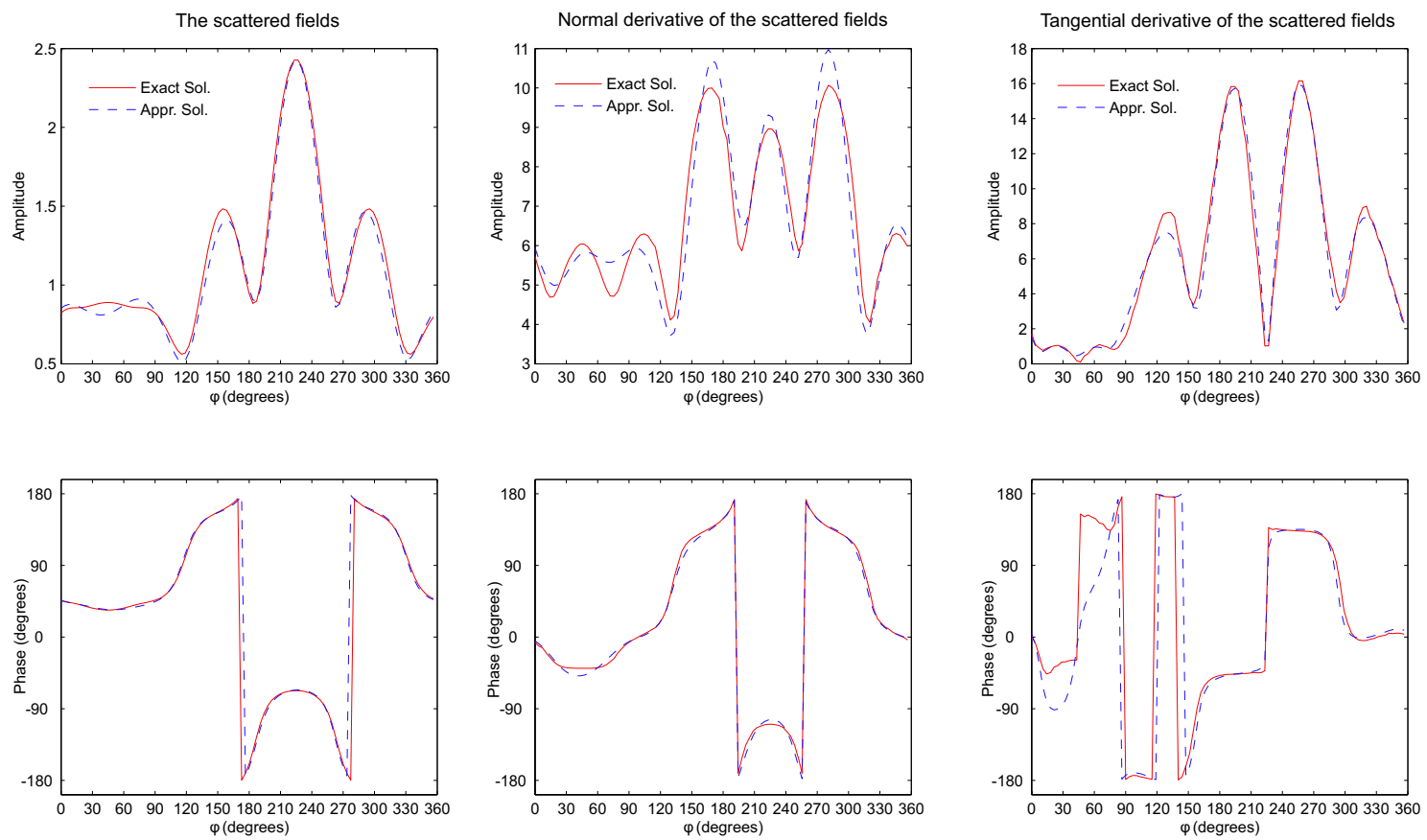

Figure 3. Comparisons of the the amplitude and the complex phase of the scattering fields and their normal and tangential derivatives on $\Gamma$ obtained by FEM for the original model and SLP for the equivalent model.

is determined by Morozov's discrepancy principle [15]. Then, for orders 1, 2, 3, the calculated fields are used in the systems of equations (2.39), (2.40) and (2.41). Here, both M and K, which are parameters related to number of the multiplier function $v_{m}$ and number of series of the width $\delta$ given in (2.37), are selected as 8 in all cases. All the integrals appearing in the abovementioned procedure are evaluated numerically by discretizing them into 100 points via the trapezoidal rule. Besides, the Newton-Raphson method is interrupted at 300 iterations except that the difference of error between two successive iterations reduces less than value $10^{-8}$.

In the first example, a circular dielectric coating with radius of $0.3 \mathrm{~m}$ is located on a PMC object whose boundary is defined in (2.12) where

$$
\delta(t)=\delta_{0}(1-0.4 \sin (6 t)) \quad t \in[0,2 \pi)
$$

with $\delta_{0}=0.1 \mathrm{~m}$ and is illuminated by 4 plane waves at $300 \mathrm{MHz}$ with incidence angles $\theta=\frac{\pi}{4}+\frac{j \pi}{2}(j=0,1,2,3)$. The dielectric permittivity and conductivity of the coating are $3 \varepsilon_{0}$ and $0.005\left(\mathrm{~S} . \mathrm{m}^{-1}\right)$, respectively. It is assumed that the scattered field $u^{s}$ is measured on the circle $\Gamma_{S}$ having radius $R=0.5 \mathrm{~m}$ for each incident wave. During the procedure of obtaining the density function $\Psi$ on the circular fictitious surface $\Gamma^{F}$ with radius $0.27 \mathrm{~m}$, Morozov's discrepancy principle determines the truncation parameter $\mathrm{N}$ as 9 for all the illuminations. The amplitude and the phase of the scattered fields and their derivatives on $\Gamma$ obtained through the FEM for the original model and the SLP for the equivalent model are compared in Figure 3 for the incident wave with angle $\theta=\frac{\pi}{4}$. As seen from the figure, the results are in a good agreement despite the data contain $10 \%$ noise. Substituting the obtained total fields and their derivatives into the system of linear equations (2.39) for 4 illuminations, the width is calculated by means of the least square method and it gives an error of about 0.25 

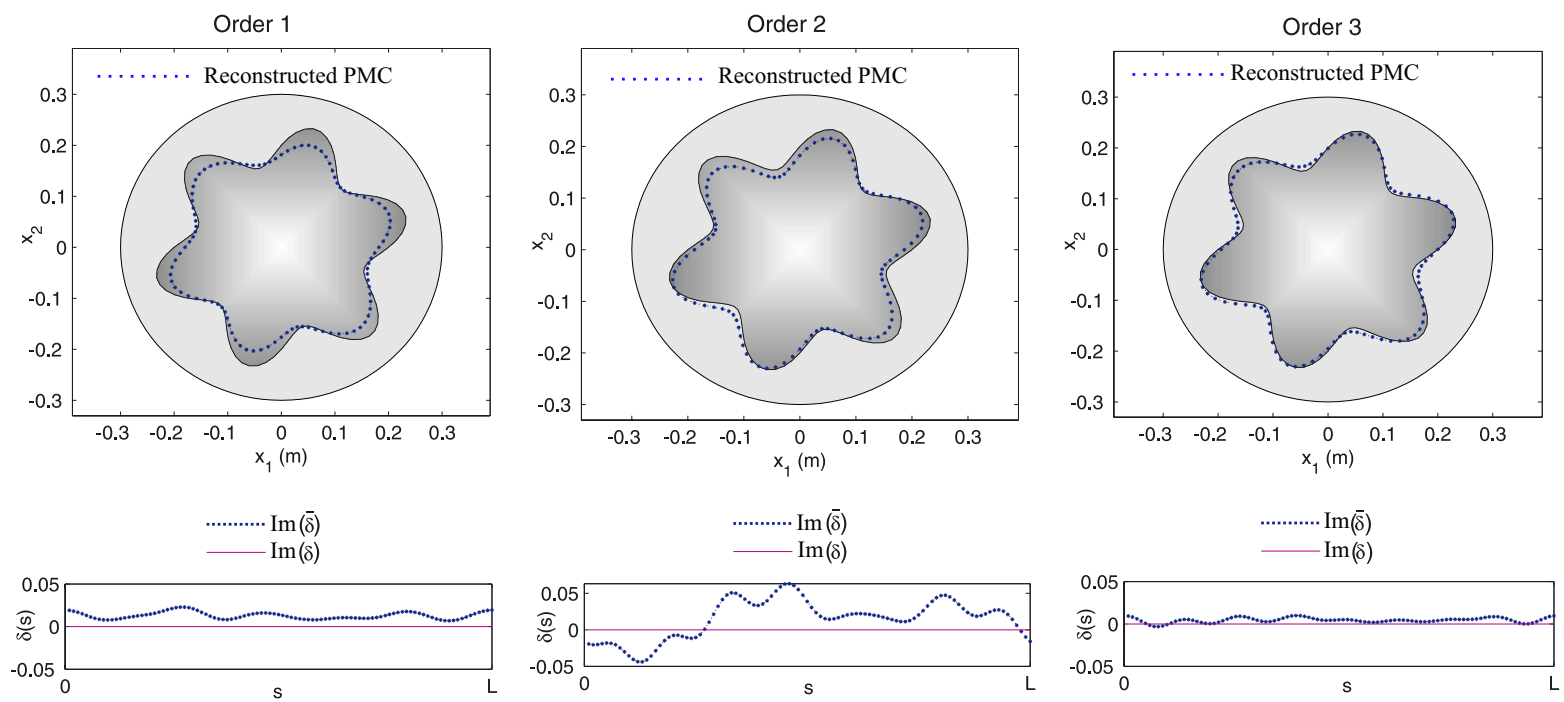

FIGURE 4. Reconstructions of the circular coating with radius $0.3 \mathrm{~m}$ and the imaginer part of the generated width $\tilde{\delta}$ for orders 1,2 and 3 .

where the error is defined as

$$
E_{r}:=\frac{\int_{\Gamma}|\delta-\tilde{\delta}| \mathrm{d} s}{\int_{\Gamma}|\delta| \mathrm{d} s}
$$

Here, $\tilde{\delta}$ symbolizes the width numerically calculated from the equivalent model. The width $\tilde{\delta}$ for orders 2 and 3 are calculated via the Newton-Raphson method by using $\tilde{\delta}$ obtained for order 1 as first initial guess. The errors for orders 2 and 3 are 0.31 and 0.08 , respectively. We note that since the approximation for the GIBC of order 1 may occasionally generate better solution than the one for the GIBC of order 2 (see [2]), here the error of order 2 is higher. The coatings, which are reconstructed through the calculated widths, are illustrated in Figure 4.

In the second example we consider a kite-shaped PMC object with the parametrization

$$
X_{\Gamma}=\left(\begin{array}{l}
0.38 \cos (t)+0.16 \cos (2 t)-0.07 \\
0.38 \sin (t)
\end{array}\right), t \in[0,2 \pi),
$$

which is coated by a circular dielectric material with radius of $0.5 \mathrm{~m}$. This coated object, whose dielectric permittivity and conductivity are respectively $2 \varepsilon_{0}$ and $0.001\left(\mathrm{~S} . \mathrm{m}^{-1}\right)$, is illuminated at $150 \mathrm{MHz}$. The synthetic data is generated on the circle $\Gamma_{S}$ having radius $R=0.8 \mathrm{~m}$ for each incident wave. The truncation parameter of TSVD inversion formula defined on the fictitious surface $\Gamma^{F}$ is assigned as 6, 7, 7, 6, 7, 7 for each illumination. Figure 5 illustrates results whose errors are respectively $0.36,0.42$ and 0.31 for orders 1,2 and 3 .

In the first two examples the curvature $c$ was chosen as a constant. As a next example, an elliptical cross-sectioned dielectric coating whose boundary is defined by

$$
X_{\Gamma}=\left(\begin{array}{c}
0.4 \cos (t) \\
0.7 \sin (t)
\end{array}\right), t \in[0,2 \pi)
$$

and the width is given by

$$
\delta(t)=0.05(\cos (2 t)-\sin (5 t)+2.2), \quad t \in[0,2 \pi),
$$



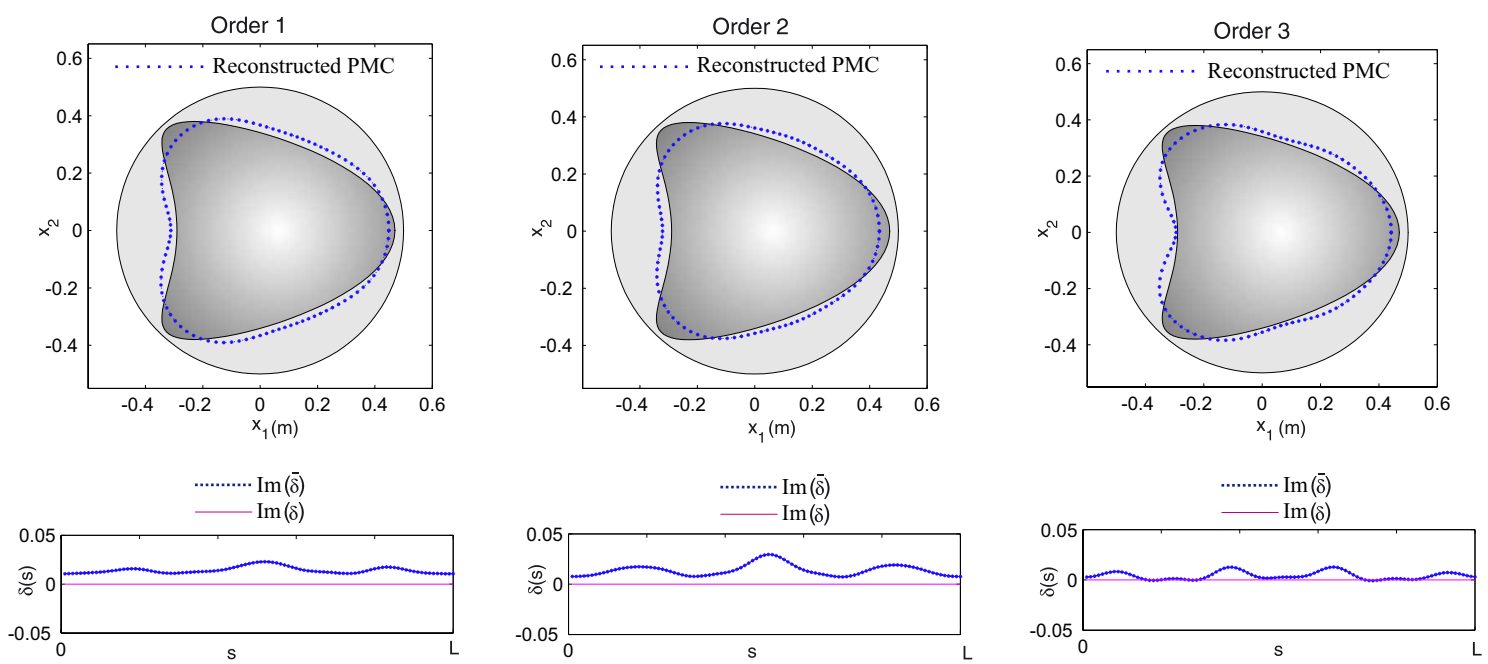

FiguRE 5. Reconstructions of the coating located on the kite-shaped PMC object and the imaginer part of the generated width $\tilde{\delta}$ for orders 1,2 and 3 .
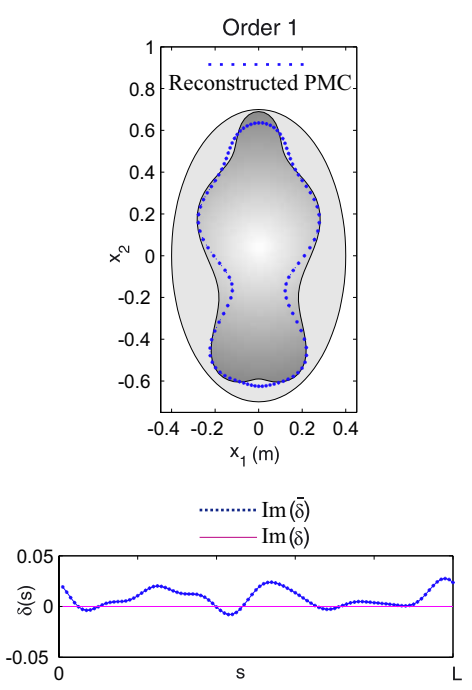
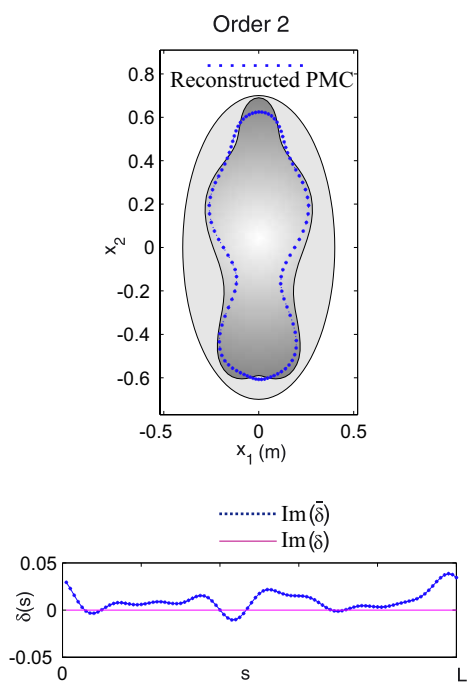
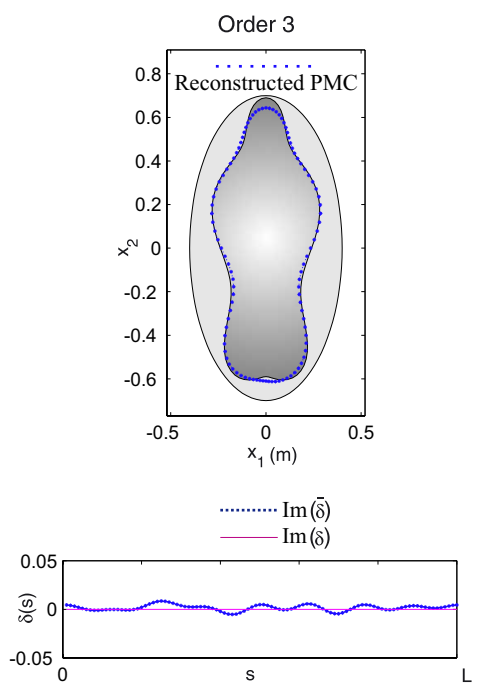

FiguRE 6. Reconstructions of the elliptical cross-sectioned coating whose boundary given in (3.4) and the imaginer part of the generated width $\tilde{\delta}$ for orders 1,2 and 3.

is taken into account. The coating is made of a material with dielectric permittivity $2.5 \varepsilon_{0}$ and conductivity

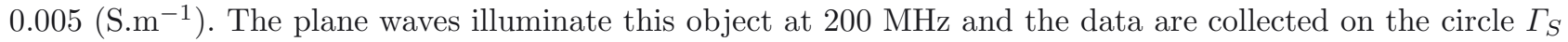
with radius $1.0 \mathrm{~m}$. The truncation parameter of the TSVD inversion formula is assigned as 9, 8, 8, 9, 11,11 for each illumination. The exact thickness and the reconstructed one for each order are shown in Figure 6 . As it is clear from the figure, the width of the coating is well determined especially for order 3 . The errors in the reconstructed widths are respectively $0.37,0.51$ and 0.27 . 

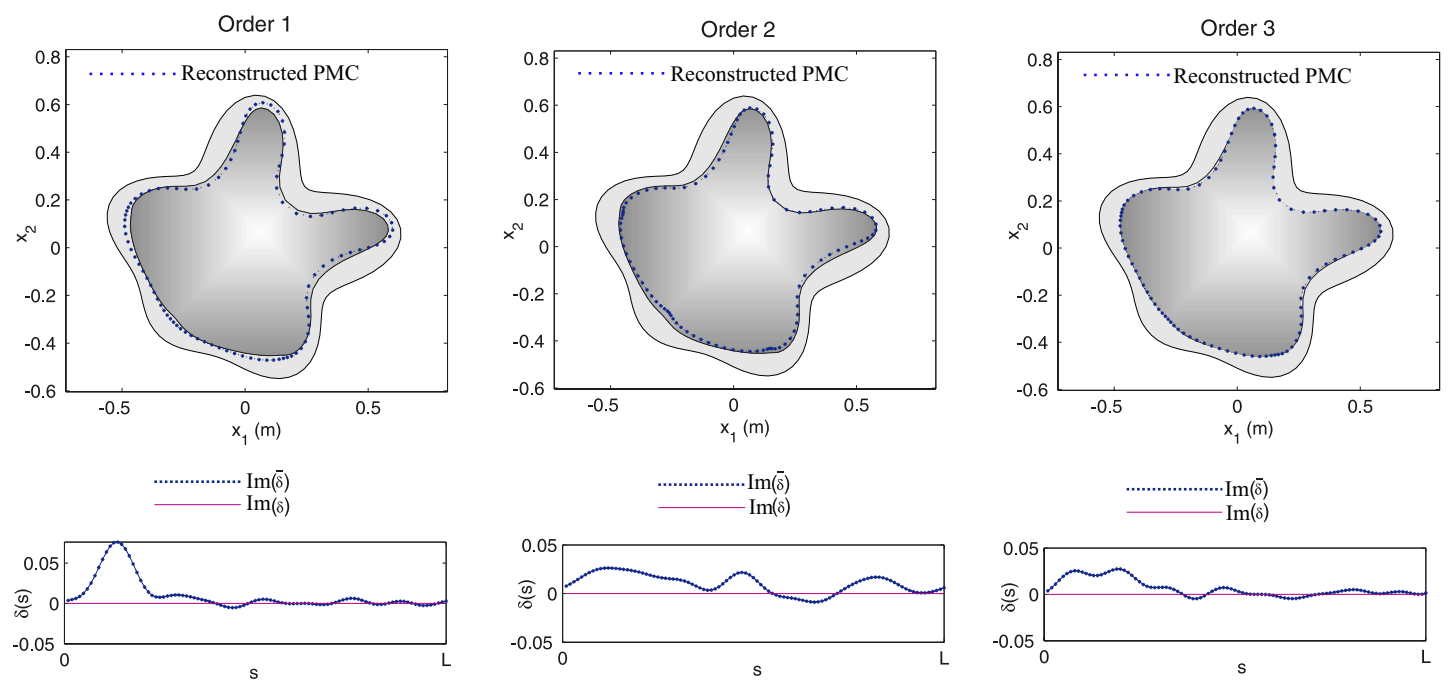

FiguRE 7. Reconstructions of the coating whose boundary given in (3.6) and width is given in (3.1) where $\delta_{0}=0.07$ and the imaginer part of the generated width $\tilde{\delta}$ for orders 1,2 and 3 .

In the next example, a more complicated coating, whose boundary is given by the parametric equation

$$
X_{\Gamma}=\left(\begin{array}{c}
(0.5+0.06 \sin (5 t)+0.07 \cos (4 t)+0.05 \cos (5 t)) \cos (t) \\
(0.5+0.06 \sin (5 t)+0.07 \cos (4 t)+0.05 \cos (5 t)) \sin (t)
\end{array}\right), t \in[0,2 \pi),
$$

and width is given in $(3.1)$ where $\delta_{0}=0.07 \mathrm{~m}$, is taken into consideration. The coating with dielectric permittivity

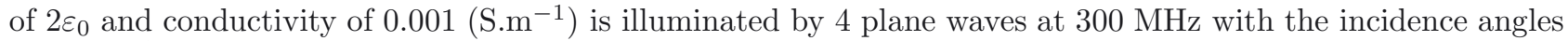
$\theta=\frac{\pi}{4}+\frac{j \pi}{2}(j=0,1,2,3)$ and the synthetic data on the circle $\Gamma_{S}$ with radius $R=0.7 \mathrm{~m}$ are calculated. The truncation parameter of the TSVD inversion formula is assigned as 15, 13, 11, 13 for each illumination. As seen in Figure 7, the results whose errors are respectively $0.28,0.27$ and 0.16 for orders 1,2 and 3 are quite satisfactory.

In order to test the validity of the approach presented here for the coating's inner boundary having corner singularities we consider two separate configurations given in Figures 8 and 9. In the first configuration a circular dielectric coating with radius of $0.5 \mathrm{~m}$ is located on a drop-shaped PMC object with the parametrization

$$
X_{\Gamma}=\left(\begin{array}{l}
0.4 \sin (t) \\
0.45+0.9 \sin (t / 2)
\end{array}\right), t \in[0,2 \pi)
$$

This coated object, whose permittivity and conductivity are $2.5 \varepsilon_{0}$ and $0.001\left(\mathrm{~S} . \mathrm{m}^{-1}\right)$, is illuminated at $200 \mathrm{MHz}$. The truncation parameters are determined as 7 for all the scattered fields which are generated on the circle $\Gamma_{S}$ with radius $1.0 \mathrm{~m}$. The reconstructed results are shown in Figure 8 . The errors in the reconstructed widths are respectively $0.16,0.15$ and 0.14 . In the second configuration a circular dielectric material with permittivity $2.5 \varepsilon_{0}$ and conductivity $0.01\left(\mathrm{~S} . \mathrm{m}^{-1}\right)$ is coated over an equilateral hexagon PMC object whose vertices are $1 \mathrm{~cm}$ away from the outer boundary. This coated object with radius $0.5 \mathrm{~m}$ is illuminated at $300 \mathrm{MHz}$ and the data are collected on the circle $\Gamma_{S}$ with radius $1.0 \mathrm{~m}$. By choosing the truncation parameters as 11, 10, 11, 12, 13, 11, which are determined by Morozov's discrepancy principle, the presented approach is applied and the relative errors are calculated as $0.25,0.43$ and 0.17 for orders 1, 2 and 3. Although the GIBCs contain higher order derivatives of the width $\delta(s)$, the proposed approach yields good reconstructions (especially for order 3 ) for the coatings having corner singularities as shown Figure 9. 

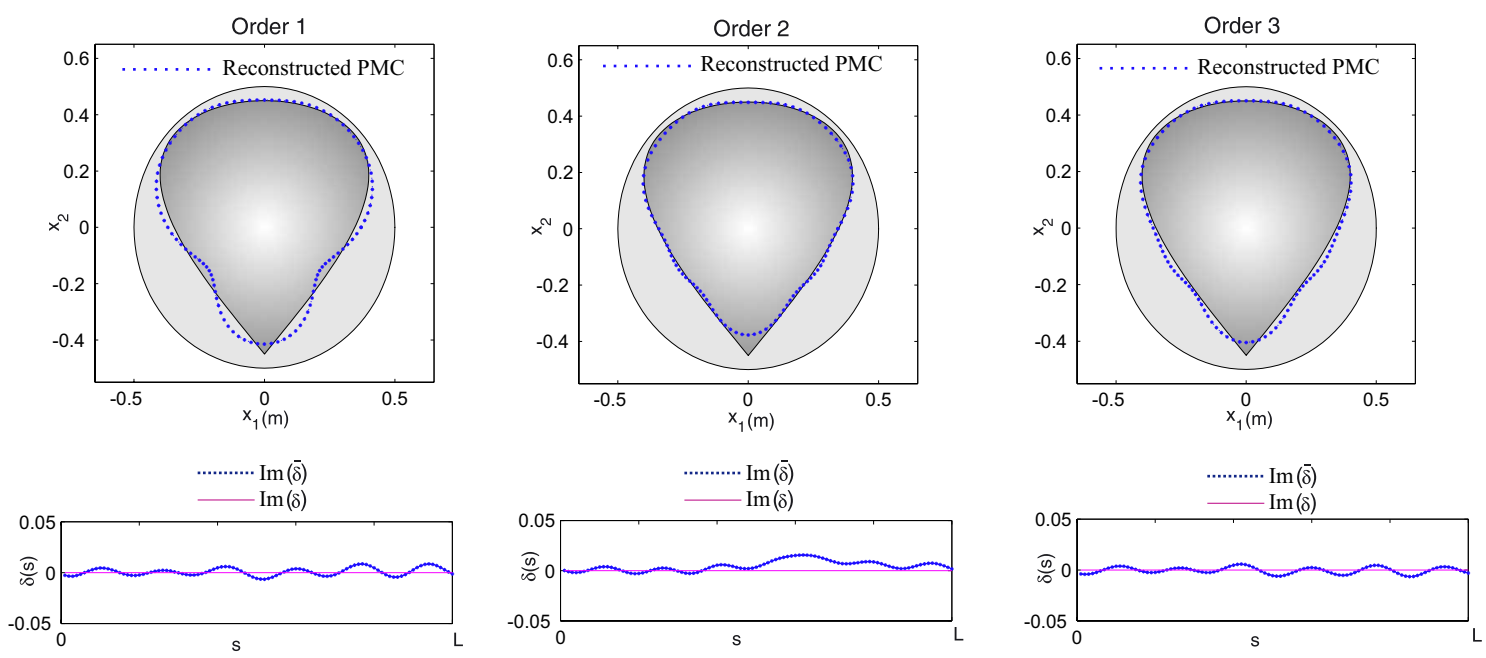

Figure 8. Reconstructions of the coating located on the drop-shaped PMC object and the imaginer part of the generated width $\tilde{\delta}$ for orders 1,2 and 3 .
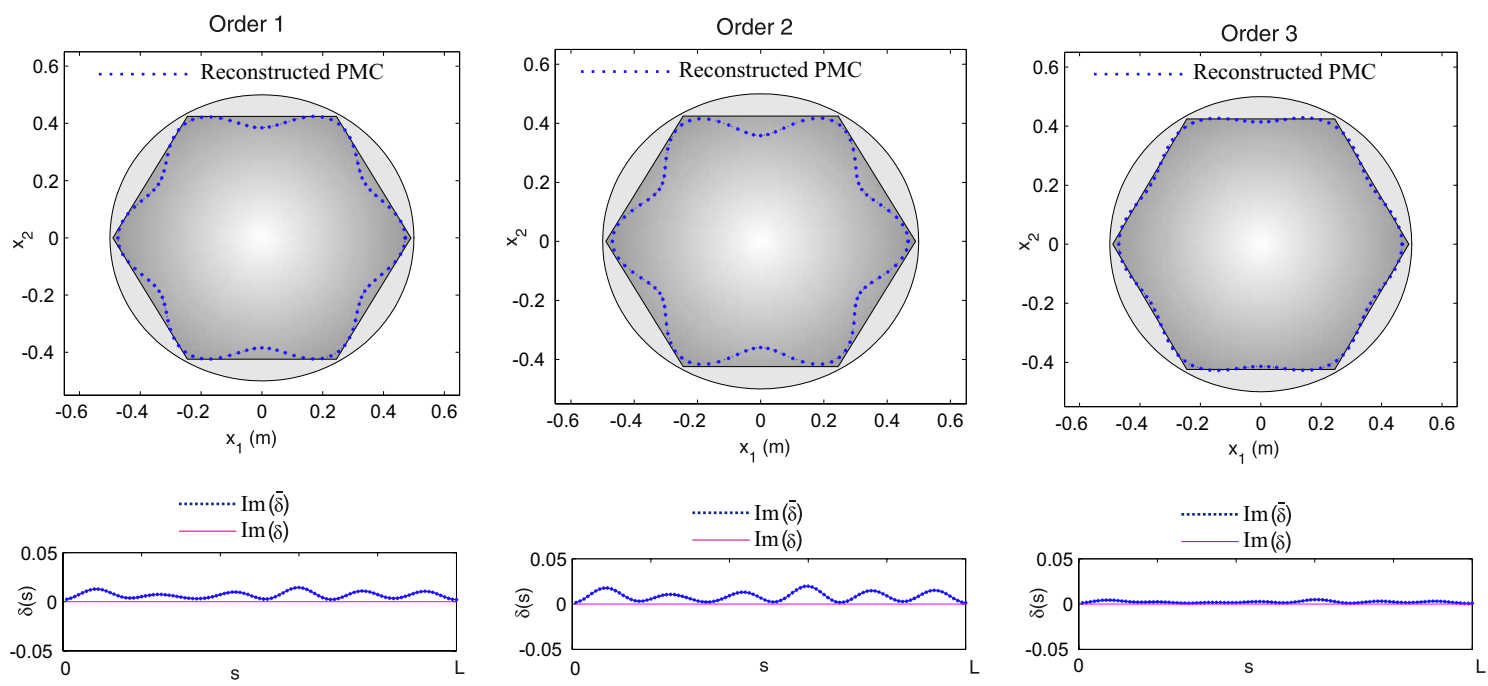

FiguRE 9. Reconstructions of the coating located on the equilateral hexagon PMC object and the imaginer part of the generated width $\tilde{\delta}$ for orders 1,2 and 3.

\section{Conclusion}

We present an approach to determine the width of the dielectric coatings having sufficiently small, variable and smooth thickness by using an equivalent model defined with help of the GIBCs that have been derived up to the third order with respect to the coating width in [2]. In this model, the GIBCs, which contain the total field and its high order normal and tangential derivatives, are defined on the outer boundary of the coating located on a PMC. In order to obtain the thickness from the expressions of the GIBCs, we first calculate the total field and its derivatives on the boundary of the coating by using a SLP approach. Then the problem is reduced to the solutions of systems of differential equations for orders 1, 2 and 3. By expanding the solution to 
a series in terms of exponential functions, the width is obtained via a solution of a system of linear equations for order 1 and systems of non-linear equations for orders 2 and 3. The non-linear systems are solved by the Newton-Raphson method.

The GIBCs are operative for the coatings whose thickness is less than one-tenth of wavelength of the incident field and conductivity is relatively small compared to the reciprocal of its width (see [2]). The numerical simulations show that the approach presented here is effective under these validity conditions of the GIBCs and robust with respect to large percentage of noise. Besides, selecting an initial guess for the Newton-Raphson method does not make difficulty due to fact that we can use the solution of the problem defined by the GIBC of order 1.

It should be lastly noted that although we handle the problem for TE polarization corresponding to a Neumann boundary condition, the approach can be applied in a similar way for TM corresponding to a Dirichlet boundary condition.

Acknowledgements. We would like to thank Houssem Haddar for his valuable advices which helped us to improve the quality of this work.

\section{REFERENCES}

[1] H. Ammari and J.C. Nedelec, Generalized impedance boundary conditions for the maxwell equations as singular perturbations problems. Commun. Partial Differ. Equ. 24 (1999) 24-38.

[2] B. Aslanyürek, H. Haddar and H. Şahintürk, Generalized impedance boundary conditions for thin dielectric coatings with variable thickness. Wave Motion 48 (2011) 681-700.

[3] L. Bourgeois, N. Chaulet and H. Haddar, Stable reconstruction of generalized impedance boundary conditions. Inverse Probl. 27 (2011) 19-38.

[4] L. Bourgeois, N. Chaulet and H. Haddar, On Simultaneous Identification of the Shape and Generalized Impedance Boundary Condition in Obstacle Scattering. SIAM J. Sci. Comput. 34 (2012) A1824-A1848.

[5] L. Bourgeois and H. Haddar, Identification of generalized impedance boundary conditions in inverse scattering problems. Inverse Probl. Imaging 4 (2010) 26.

[6] M. Cayoren, I. Akduman, A. Yapar and L. Crocco, A new algorithm for the shape reconstruction of perfectly conducting objects. Inverse Probl. 23 (2007) 1087-1100.

[7] R. Cicchetti, A class of exact and higer-order surface boundary conditions for layered structures. IEEE Trans. Antennas Propag. 44 (1996) 249-259.

[8] D. Colton and R. Kress, Inverse Acoustic and Electromagnetic Scattering Theory, 2nd edn. Springer, Berlin (1999).

[9] M. Duruflé, H. Haddar and P. Joly, Higher Order Generalized Impedance Boundary Conditions in Electromagnetic Scattering Problems. C.R. Phys. 7 (2006) 533-542.

[10] H. Haddar, P. Joly and H.M. Nguyen, Generalized impedance boundary conditions for scattering by strongly absorbing obstacles: the scalar case. Math. Models Methods Appl. Sci. 15 (2005) 1273-1300.

[11] H. Haddar, P. Joly and H.M. Nguyen, Generalized impedance boundary conditions for scattering problems from strongly absorbing obstacles: the case of Maxwell's equations. Math. Models Methods Appl. Sci. 18 (2008) 1787-1827.

[12] H. Haddar and A. Lechleiter, Asymptotic models for scattering from unbounded media with high conductivity. Math. Mod. Numer. Anal. 44 (2010) 1295-1317.

[13] L. He, S. Kindermann and M. Sini, Reconstruction of shapes and impedance functions using few far-field measurements. J. Comput. Phys. 228 (2009) 717-730.

[14] J.L. Holloway, E.F. Kuester, Impedance-type boundary conditions for a periodic interface between a dielectric and a highly conducting medium. IEEE Trans. Antennas Propag. 48 (2000) 1660-1672.

[15] J. Kaipio and E. Somersalo, Statistical and Computational Inverse Problems 1st edn. Springer, New York (2004).

[16] I. Knowles, A variational algorithm for electrical impedance tomography. Inverse Probl. 14 (1998) $1513-1525$.

[17] I. Knowles, Parameter identification for elliptic problems. J. Comput. Appl. Math. 131 (2001) 175-194.

[18] M.J. Kong and B. Beker, Computation of resonant frequencies of cylindrical ferrite resonators using GIBCs. IEEE Trans. Microwave Theory Tech. 46 (1998) 1503-1507.

[19] J.J. Liu, G. Nakamura and M. Sini, Reconstruction of the shape and surface impedance from acoustic scattering data for an arbitrary cylinder. SIAM J. Appl. Math. 67 (2007) 1124-1146.

[20] M. Ljalinov, Generalized impedance boundary conditions and model of a point conductor. Radio Sci. 30 (1995) $1777-1786$.

[21] O. Marceaux and B. Stupfel, Higher order impedance boundary conditions for multilayer coated 3-D objects. IEEE Trans. Antennas Propag. 46 (2000) 429-436.

[22] O. Ozdemir, I. Akduman, A. Yapar and L. Crocco, Higher order inhomogeneous impedance boundary conditions for perfectly conducting objects. IEEE Trans. Geosci. Remote Sens. 45 (2007) 1291-1297. 
[23] O. Ozdemir, H. Haddar and A. Yaka, Reconstruction of the electromagnetic field in layered media using the concept of approximate transmission conditions. IEEE Trans. Antennas Propag. 59 (2011) 2964-2972.

[24] H.Y. Pao, Z. Zhu and S.L. Dvarok, Exact, closed-form representation for the time-domain surface impedances of a homogeneous, lossy half-space. IEEE Trans. Antennas Propag. 52 (2004) 2659-2665.

[25] J.R. Poirier, A. Bendali and P. Borderies, Impedance boundary conditions for the scattering of time-harmonic waves by rapidly varying surfaces. IEEE Trans. Antennas Propag. 54 (2006) 995-1005.

[26] Z.G. Qian, W.C. Chew and R. Suaya, Generalized impedance boundary condition for conductor modeling in surface integral equation. IEEE Trans. Microwave Theory Tech. 55 (2007) 2354-2364.

[27] Z.G. Qian, M.S. Tong and W.C. Chew, Conductive medium modeling with an augmented GIBC formulation. PIER 99 (2009) $261-272$.

[28] C.R. Rao, Linear Statistical Inference and Its Applications 2nd edn. Wiley, New York (1973).

[29] R.G. Rojas, Generalized impedance boundary conditions for EM scattering problems. Electron Lett. 24 (1998) $1093-1094$.

[30] R.G. Rojas, Z. Al-hekail, Generalized impedance/resistive boundary conditions for EM scattering problems. Radio Sci. 24 (1989) $1-12$

[31] K. Schmidt and S. Tordeux, High order transmission conditions for thin conductive sheets in magneto-quasistatics. Math. Mod. Numer. Anal. 45 (2011) 1115-1140.

[32] T.B.A. Senior, J.L. Volakis and S.R. Legault, Higher order impedance and absorbing boundary conditions. IEEE Trans. Antennas Propag. 45 (1997) 107-114.

[33] M. Yousefi, S.K. Chaudhuri and S. Safavi-Naeini, GIBC formulation for the resonant frequencies and field distribution of a substrate-mounted dielectric resonator. IEEE Trans. Antennas Propag. 42 (1994) 38-46.

[34] S. Yuferev, L. Proekt and N. Ida, Surface impedance boundary conditions near corners and edges: Rigorous consideration. IEEE Trans. Magn. 37 (2001) 3466-3468. 